\title{
Video Article \\ Paw-Print Analysis of Contrast-Enhanced Recordings (PrAnCER): A Low- Cost, Open-Access Automated Gait Analysis System for Assessing Motor Deficits
}

\author{
Hayley A. Bounds ${ }^{1}$, Devon L. Poeta ${ }^{1}$, Petra M. Klinge ${ }^{3}$, Rebecca D. Burwell ${ }^{1,2}$ \\ ${ }^{1}$ Cognitive, Linguistic \& Psychological Sciences, Brown University \\ ${ }^{2}$ Department of Neuroscience, Brown University \\ ${ }^{3}$ Department of Neurosurgery, Warren Alpert Medical School, Brown University
}

Correspondence to: Rebecca D. Burwell at rebecca_burwell@brown.edu

URL: https://www.jove.com/video/59596

DOI: doi:10.3791/59596

Keywords: Behavior, Issue 150, gait analysis, paw prints, motor impairment, neurodegenerative disease, Parkinson's Disease, pain, nerve damage, rodent

Date Published: 8/12/2019

Citation: Bounds, H.A., Poeta, D.L., Klinge, P.M., Burwell, R.D. Paw-Print Analysis of Contrast-Enhanced Recordings (PrAnCER): A Low-Cost, Open-Access Automated Gait Analysis System for Assessing Motor Deficits. J. Vis. Exp. (150), e59596, doi:10.3791/59596 (2019).

\section{Abstract}

Gait analysis is used to quantify changes in motor function in many rodent models of disease. Despite the importance of assessing gait and motor function in many areas of research, the available commercial options have several limitations such as high cost and lack of accessible, open code. To address these issues, we developed PrAnCER, Paw-Print Analysis of Contrast-Enhanced Recordings, for automated quantification of gait. The contrast-enhanced recordings are produced by using a translucent floor that obscures objects not in contact with the surface, effectively isolating the rat's paw prints as it walks. Using these videos, our simple software program reliably measures a variety of spatiotemporal gait parameters. To demonstrate that PrAnCER can accurately detect changes in motor function, we employed a haloperidol model of Parkinson's disease (PD). We tested rats at two doses of haloperidol: high dose $(0.30 \mathrm{mg} / \mathrm{kg})$ and low dose $(0.15 \mathrm{mg} / \mathrm{kg})$. Haloperidol significantly increased stance duration and hind paw contact area in the low dose condition, as might be expected in a PD model. In the high dose condition, we found a similar increase in contact area but also an unexpected increase in stride length. With further research, we found that this increased stride length is consistent with the bracing-escape phenomenon commonly observed at higher doses of haloperidol. Thus, PrAnCER was able to detect both expected and unexpected changes in rodent gait patterns. Additionally, we confirmed that PrAnCER is consistent and accurate when compared with manual scoring of gait parameters.

\section{Video Link}

The video component of this article can be found at https://www.jove.com/video/59596/

\section{Introduction}

Rodents are commonly used as models to study a wide range of diseases and injuries including arthritis ${ }^{1}$, Parkinson's Disease (PD) $)^{2,3}$, neuromuscular disorders ${ }^{4,5}$, hydrocephalus ${ }^{6}$, and spinal cord injury ${ }^{7}$. In these conditions, symptoms such as pain, balance, and motor function can be measured by studying the animals' gait patterns. These patterns are quantified using a set of spatiotemporal gait parameters that summarize the location and timing of paw prints as well as the area of paw contact on the ground.

Although many options for gait analysis exist, current systems have several drawbacks. In traditional ink and paper testing, an animal's paws are coated with ink before it walks across a sheet of white paper (Figure 1A). The resulting paw prints can then be measured for stride length and stance width, but key temporal gait parameters such as speed or step duration cannot be assessed. Modern video-based systems are more reliable, but video analysis requires laborious frame-by-frame scoring unless a suitable automated system is used ${ }^{8}$. There are many commercial automated scoring systems currently available, but these systems can be prohibitively expensive. Additionally, these systems rely on clear flooring or in some cases, treadmills, both of which alter natural movement. Treadmills have been shown to mask motor deficits in some disease models $^{9}$, while clear flooring (Figure 1B) causes mice to spend more time on the perimeter of an open field, indicating increased anxiety ${ }^{10}$. Ideally, a gait analysis apparatus would not rely on either, producing the most natural movement patterns with the least stress to the animal.

Available open-source and commercial options use a variety of methods to overcome the difficulty of isolating a footprint from the animal's body despite variable lighting conditions, animal color, and print shapes. Some enhance the contrast of contacting paws using surfaces that release light in response to pressure ${ }^{7,11,12}$, but these are expensive and technically difficult to construct. Other systems utilize multiple view angles which permit observation of whole-body coordination ${ }^{8,13}$. While these options offer advantages for measuring additional motor parameters beyond gait, they are unnecessarily complex for simple gait analysis. Further, all of these techniques rely on clear flooring, which alters natural behavior.

PrAnCER is based on what we call Contrast-Enhanced Recordings, which use a combination of lighting and a semitransparent floor to enhance the detection of prints. When viewed from below, this creates a high-contrast image (paw print) while obscuring the view of objects not in contact 
with the surface (the animal's body) (Figure 1D). When viewed from above, the floor appears opaque. The resulting salience of the paws in our method permits accurate identification of a variety of gait and locomotor characteristics by our newly developed automated system. In the present study, we describe the apparatus, our gait analysis protocol, and our automated scoring system, PrAnCER. Our apparatus is easily assembled and PrAnCER can be used to assess motor deficits in a wide range of disease and injury models.

To demonstrate that PrAnCER can be used to detect abnormal gait patterns, we used a haloperidol model of PD, a simple model for transient induction of locomotor changes ${ }^{14}$. Haloperidol is a dopamine receptor antagonist widely used as an antipsychotic ${ }^{1}$. It affects motor systems by altering dopamine signaling in the striatum, an important component of the motor pathway in the basal ganglia ${ }^{14}$. Even a single dose of haloperidol rapidly reduces extracellular dopamine levels in the striatum, causing Parkinsonian-like motor defecits ${ }^{15}$. The behavioral effects are muscular rigidity, akinesia, and catalepsy, which is defined as an inability to return to a normal posture after being placed in an unusual position ${ }^{11,16}$. Acute doses of haloperidol cause locomotor deficits identifiable in the rotarod test of motor function ${ }^{17}$. We reasoned that haloperidolmediated locomotor impairments would also be evident in a number of characteristics accessible to automated gait analysis.

Although responses to haloperidol vary widely across studies, cataleptic effects of haloperidol emerge at doses of $0.5 \mathrm{mg} / \mathrm{kg}$ and higher, while reduced responsiveness and motor impairment is detectable at lower doses $(0.1-0.3 \mathrm{mg} / \mathrm{kg})^{16,17}$. In an effort to avoid the cataleptic effects of haloperidol, we decided to test two doses of haloperidol: a high dose $(0.30 \mathrm{mg} / \mathrm{kg})$ and a low dose $(0.15 \mathrm{mg} / \mathrm{kg})$. As shown in Table 1, Experiment 1 examined the effects of high dose haloperidol, while Experiment 2 tested the effects of low dose haloperidol. We used a within-subject design in which every rat was tested in the high dose, low dose, and control (saline) conditions. The order of condition was counterbalanced across rats. We predicted that acute administration of haloperidol would cause gait impairments similar to those found in other models of PD such as decreased speed, decreased stride length, and longer stance duration ${ }^{3,14,18,19}$. We observed behavioral changes including akinesia following haloperidol administration at both dosages. In the low dose condition, rats had significantly increased stance duration and hind paw contact area, as expected. These gait changes are comparable to the slow, shuffling steps common among PD patients ${ }^{2,20}$. In the high dose condition, however, we saw an increase in stride length as well as an increase in paw contact area. Although the increase in stride length was unexpected, further review of the literature indicated that it is likely part of a haloperidol-induced bracing-escape response. We conclude that PrAnCER is indeed capable of detecting Parkinsonian-like changes in rodent gait consistent with the use of neuroleptics.

\section{Protocol}

All procedures were in accordance with Brown University Institutional Animal Care and Use Committee guidelines.

\section{Gait analysis apparatus}

1. Prepare the gait analysis walkway consisting of a clear plexiglass enclosed walkway ( 36 " $L \times 3$ " W $\times 4.5$ " $H$ ) placed on a clear plexiglass floor (Figure 2A). Make the plexiglass floor semitransparent by covering it with a piece of 16 LB cotton fiber drafting vellum cut to the same width as the walkway.

NOTE: There are other methods for making the floor semitransparent.

2. Place a camera with a frame rate of at least 30 frames per second (fps) directly below the walkway to capture the middle of the track (Figure 2B).

3. Secure a strip of $12 \mathrm{~V}$ LED lights with $18 \mathrm{LEDs} /$ foot approximately 2 inches away from and 1 inch above the floor of the walkway to illuminate the track.

\section{Animal preparation}

1. Allow the animals to acclimate to the vivarium for at least 1 week prior to handling. Handle the rats for at least 5 days prior to beginning experiment. This study used 8 male Long Evans rats approximately 3 months old.

2. Habituate the animals to the testing room and gait walkway with the room lights turned off

1. Place the rat's home cage at the surface level at the end of the gait walkway to serve as a goal box. Note that if the home cage is deep, aged or locomotor-impaired rats may benefit from a ramp or step to provide easier access to the home cage.

2. Allow the rat to walk from the experimenter's hand down the length of the walkway to reach its home cage.

3. Rats will often stop at the end of the walkway to look around before jumping down into the home cage. If a rat takes longer than 1 min to exit the walkway, encourage it to enter its home cage with a gentle push.

4. If the rat turns around, use a small piece of plexiglass to block the "start" end of the walkway. Repeat for a total of 3 runs.

3. Habituate for at least 2 days or until the rats are comfortable crossing the walkway at a steady pace without freezing

\section{Gait testing procedure}

1. Adjust the settings on the webcam software to achieve the clearest picture of the paw prints. Turn off room lights for all gait testing.

2. Record each run separately and label appropriately for use with the automated analysis program.

3. Ensure there are no spots or debris on the vellum. Begin recording a few seconds before the rat enters the walkway and stop once the rat exits the walkway and enters its home cage.

4. Continue until either three acceptable runs are completed or 10 min has elapsed.

5. Wipe down the walkway with ethanol in between each rat and replace the vellum as needed.

NOTE: An acceptable trial is defined as one in which the animal walks consistently and without pause for the first 4 steps of the run. If this is hard to achieve, adjust the criteria to include trials in which there are 4 consecutive steps at any point in the run that occur without pauses or abrupt acceleration. 


\section{PrAnCER automated analysis}

1. Put all videos to be analyzed in a folder.

2. Launch PrAnCER by running the Python script PrAnCER. PrAnCER will analyze the videos based on the steps illustrated in Figure 3 and Figure 4.

3. In the pop-up menu, select the specified folder by pressing the Choose a folder button. Select custom options for analysis if desired. Detailed descriptions of each parameter can be found by clicking the question mark next to them. Click Continue when finished.

4. Define a region of interest (ROI) on the image of the walkway that appears. To do this, left click to define a top edge and right click to define a bottom edge. If the box that appears is correct, press $\mathbf{N}$ to continue. If not, press $\mathbf{Z}$ to undo. Once $\mathbf{N}$ is pressed, the program will run automatically.

5. After PrAnCER is complete, terminate the program by pressing Enter in the terminal.

6. To manually review the results output by PrAnCER, run the Python script GaitEditorGUI and select the appropriate .mp4 file for each video. If needed, correct any mis-identified or merged prints.

7. To extract spatial and temporal gait parameters, run the Python script ParameterAnalyzer. Choose the number of hind prints to analyze and the folder of videos to analyze, then click Continue. This will output a .csv file for each video containing a number of common gait parameters, which are described in Table 2 and illustrated in Figure 5.

NOTE: The full scripts, as well as instructions for reading and analyzing data, are available at the author's GitHub (www.github.com/ hayleybounds). We implemented this algorithm using the free, open-source Python library OpenCV ${ }^{21}$. Also included on the GitHub are instructions for building our gait analysis walkway.

\section{Representative Results}

\section{Haloperidol procedure}

We developed this gait analysis system to compare gait parameters in control rats to those in experimental rats expected to show a variety of locomotor, gait, and balance impairments. We used a within-subject design in which every rat was tested in the saline, high dose haloperidol and low dose haloperidol conditions. Rats were separated into two groups ( $\mathrm{A}$ and $\mathrm{B}$ ) to allow counterbalancing; gait testing was counterbalanced for time of day and order of condition. Each test was separated by $48 \mathrm{~h}$. Rats were lightly anesthetized with isoflurane before receiving intraperitoneal (IP) injections of either saline or haloperidol. Gait was tested $1 \mathrm{~h}$ post-injection, at which point the haloperidol should be at peak levels ${ }^{15,16,17}$.

\section{Behavioral results}

We observed prominent behavior changes in animals treated with haloperidol. In the high dose condition, five of the eight rats had periods of immobility at the start of the walkway, during which they were unresponsive to the experimenter touching them and resistant to being moved. In some cases, this state persisted for several minutes until the rat was removed from the walkway. In other cases, the immobile rat would suddenly move rapidly or "bound" across the walkway and then return to the immobile state near the end. In the low dose condition, 3 of the 8 rats had similar periods of immobility. At this dosage, there was only one instance of bounding behavior. No bounding was observed when the animals were treated with saline.

We analyzed the effects of haloperidol on the following gait parameters: base of support, stride length, stride speed, stance duration, stance to swing ratio, maximum contact area, and interlimb distance. Because many gait parameters for front and hind limbs are identical and haloperidol generally has uniform impacts on all limbs, we calculated parameters for only the hind limbs and did not separate data for left and right limbs. For each rat, we calculated the mean of each gait parameter from all usable runs from each testing day. All parameters (other than speed variability) were calculated as the mean for the first 4 usable steps of a run. To assess whether each dosage of haloperidol significantly impacted gait, we used a paired sample t-test. In Experiment 1, there was a significant increase in stride length (Figure $6 \mathbf{A} ; t(7)=-2.962, p=0.021)$ and maximum contact area (Figure 6A; $t(7)=-2.51, p=0.04$ ) in animals treated with high dose haloperidol. Base of support, speed, stance duration and stance to swing ratio were not significant. In Experiment 2, animals given low dose haloperidol showed a significant increase in stance duration (Figure 6B; $t(7)=-2.444, p=0.044$ ) and maximum contact area (Figure 6B; $t(7)=-3.085, p=0.018$ ) compared to the saline condition. No other gait parameters were significant. Additionally, there was a significant difference between the high dose and low dose haloperidol conditions in base of support (Figure $6 \mathrm{C} ; \mathrm{t}(7)=2.651, \mathrm{p}=0.033$ ), maximum contact area (Figure $6 \mathrm{C} ; \mathrm{t}(7)=4.635, \mathrm{p}=0.002)$ and interlimb distance (Figure $6 C ; t(7)=3.098, p=0.017)$

\section{Your location accuracy and errors in the automated system}

To assess the accuracy of PrAnCER, we compared its automated analysis to the manual scoring of 21 randomly selected videos from a separate group of 6 control rats. For hand scoring purposes, the videos were converted into a sequence of images, which were then used to manually mark the locations of prints. For efficiency, we focused our analysis on spatial data measured from hind prints only. We extracted the mean stride length and BOS for each video and compared it to the automated values. While mean stride length was not significantly different between manual scoring and PrAnCER analysis (Figure 7B; $\mathrm{t}(20)=-0.01, \mathrm{p}=0.99$ ), base of support was significant $($ Figure 7A; $\mathrm{t}(20)=-2.21, \mathrm{p}=0.038)$. Though the automated and manual scoring was generally well correlated, the automated system reported a $5 \%$ larger BOS on average. This difference may be due to variances in centroid selection rather than detection errors. For manual scoring, the print location was marked drawing an oval around the base of each hind print, as it would be difficult to manually replicate PrAnCER's method of center of mass estimation. The clear trend was for PrAnCER to overestimate BOS, perhaps because some animals may splay their toes out in an asymmetric fashion, causing PrAnCER to observe more extreme centroids than manual scoring. Other systems have also noted significant increases in BOS between manual and automated scoring despite consistent stride length measures ${ }^{17}$. Considering the small differences observed and the consistency with other systems, we conclude that PrAnCER is a reliable measure of gait parameters. 
It is important to note that all accuracy analysis occurred after manual correction of the automated output was performed using PrAnCER's GUI As in existing commercial systems, this step is necessary both for correcting errors in scoring and for eliminating runs that do not meet criteria ${ }^{22}$. We tuned PrAnCER to err on the side of false positives, as these are easier to correct post-hoc. We have never observed PrAnCER fail to detect a real print during manual correction of over 5 hundred videos. Other types of errors, however, were observed. These fell into 3 categories: false detections (detection of a non-print as a print), misclassifications (print mislabeled as front/hind or left/right), and false combinations (two prints incorrectly merged). These errors are easily corrected in the accompanying GUI, and typically occur in only a small percentage of videos filmed under normal conditions. Even with such corrections, PrAnCER markedly decreases the amount of manual labor involved in gait analysis. We estimate that for each video it takes approximately $3 \mathrm{~min}$ to run PrAnCER and correct any output errors (if necessary), while it would take nearly 10 min to manually score and analyze the same video.
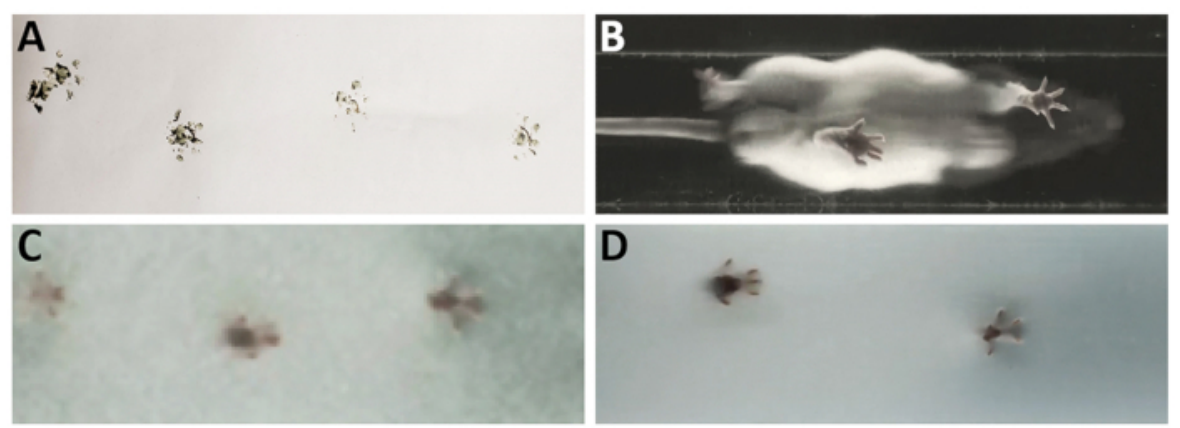

Figure 1. Comparison of gait analysis methods. (A) The traditional ink and paper method produces imprecise prints of paw shape and location. (B) Video recording with a transparent floor gives a detailed view of the paw prints but contains many salient features from the rat's body that complicates automated scoring. (C) Lightweight paper over a clear floor creates a noisy image and loses details. (D) The use of vellum to create a translucent floor produces highly detailed prints while visually eliminating the body. Please click here to view a larger version of this figure.

A

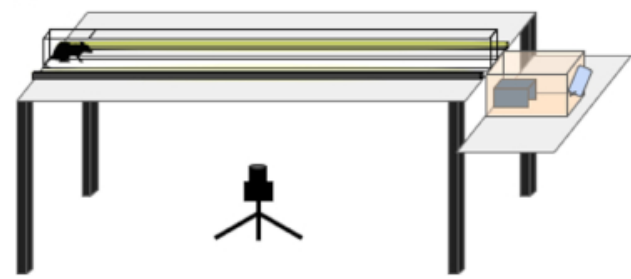

B

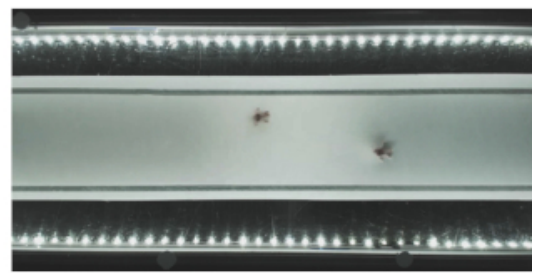

Figure 2. Schematic illustration of the gait walkway apparatus and video recording. (A) The rat walks through a clear walkway with a translucent floor to the home cage goal box while being recorded from below. In this case, the vellum covers a transparent floor to make it translucent. The walkway is illuminated by LED strips placed along its length at a level between the animal's feet and body. (B) A screenshot of a video recording demonstrating the effects of the translucent floor. Two paws are clearly visible, but the rat's body is essentially undetectable. Please click here to view a larger version of this figure.

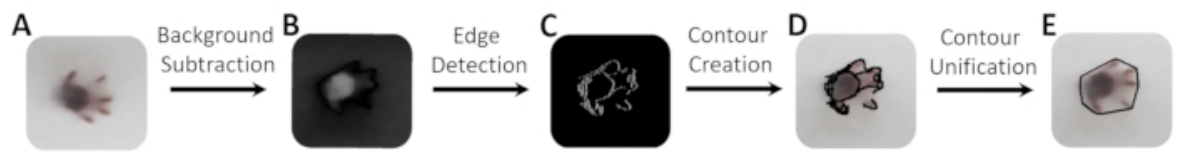

Figure 3. Detection process for one frame of a paw print. (A) The original image is de-noised and then subjected to background subtraction (B). (C) An edge detection algorithm is applied and the results are converted to a series of $X, Y$ coordinates called contours (D). (E) Contours are grouped by proximity and the convex hull (bounding box) of the group is taken to produce a single contour encompassing the print. Please click here to view a larger version of this figure. 
A
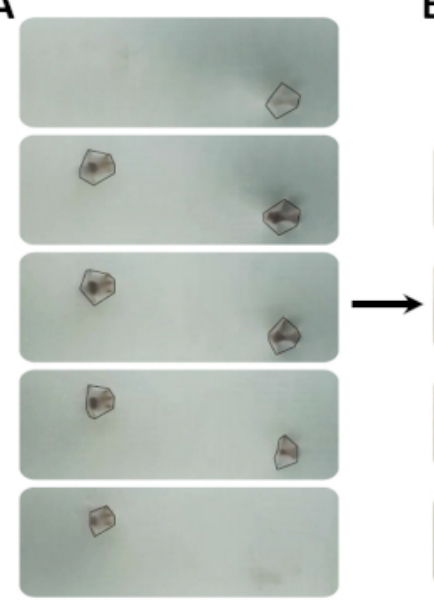

B

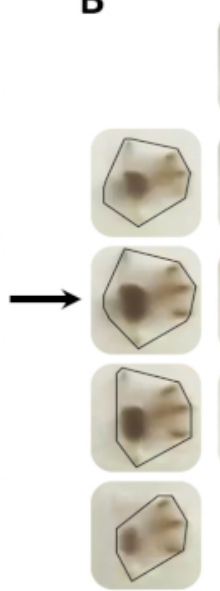

C
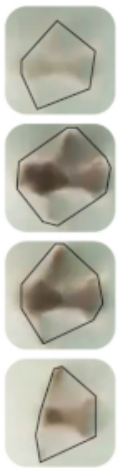

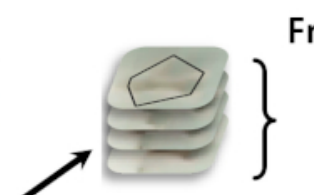

\section{Front Right}

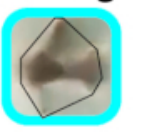

Hind Left
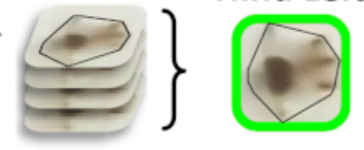

Figure 4. Conversion of individual detections into a classified print. (A) Paw prints are first identified in a set of frames. (B) Individual object detections are given a number that identifies them as a print, representing a single placement of one paw (C). (D) Finally, they are classified as left or right based on their location relative to the midline of the animal's path, and front or hind based on their location relative to the previous paw prints. Please click here to view a larger version of this figure.

A
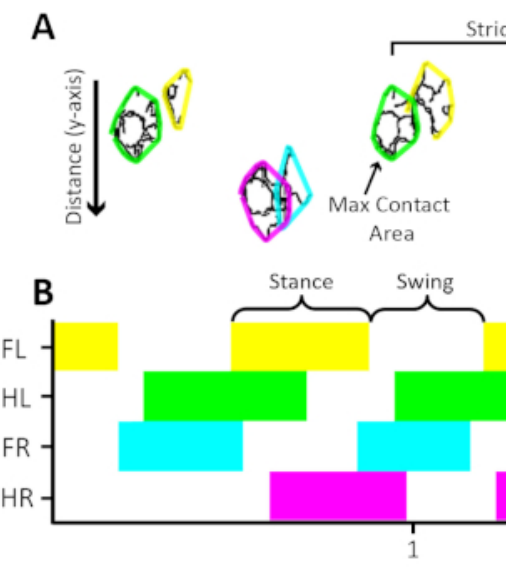

Stride Length

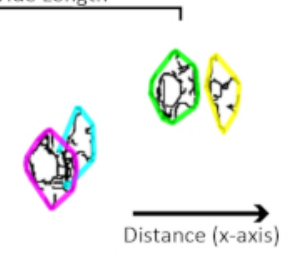

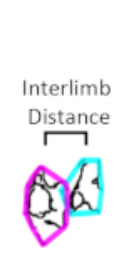

nterlimb Distance

istance ( $\mathrm{x}$-axis

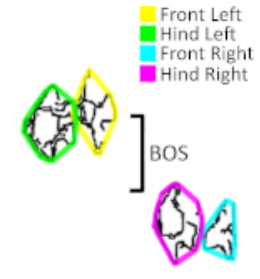

$2 \mathrm{~cm}$

Figure 5. Illustration of gait parameters analyzed. (A) An example output showing the identification and location of paw prints. Original detected edges are shown in black. Final detected paws and approximate area are shown in colors that indicate paw classification. In this figure, yellow: front left, green: hind left, cyan: front right, and magenta: hind right. However, the colors can be changed in the Python script according to user preference. (B) A plot illustrating two major temporal parameters: the amount of time each paw is in contact with the ground (stance phase) and in the air (swing phase). Colored blocks indicate the stance phase and the white spaces indicate the swing phase. Please click here to view a larger version of this figure. 
A
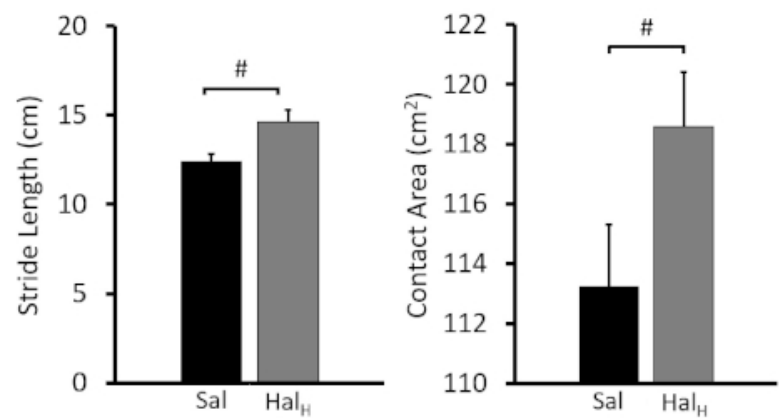

B
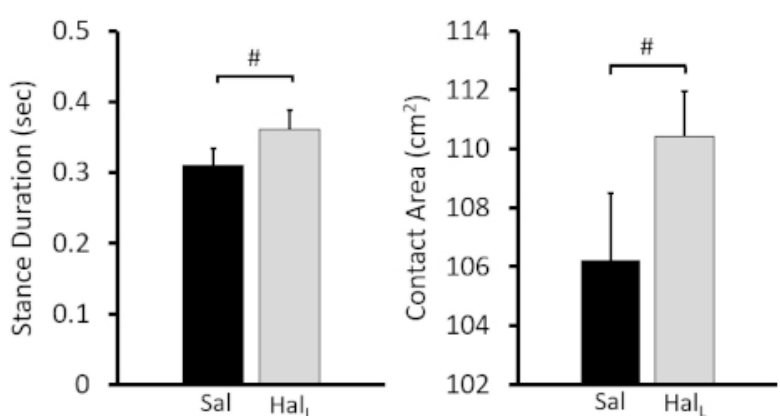

C
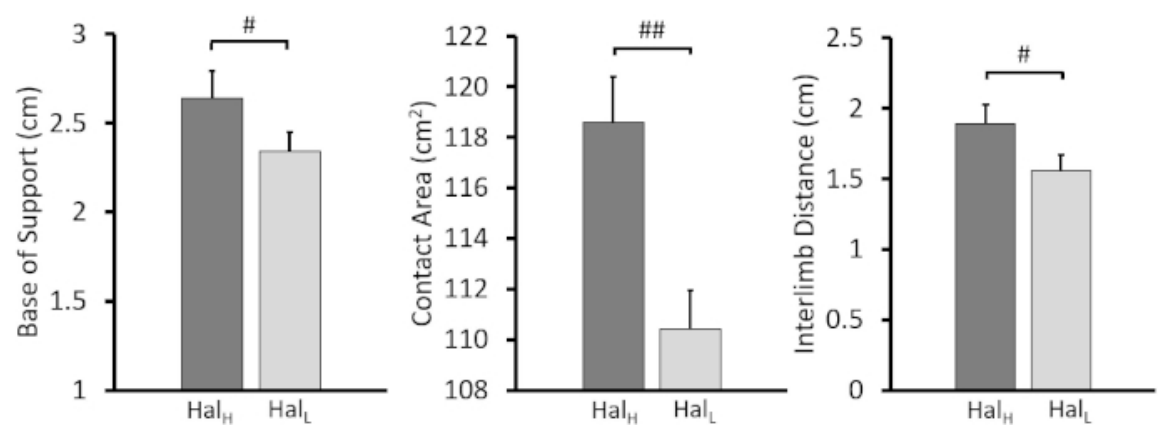

Figure 6. Effects of haloperidol on gait. (A) Results of Experiment 1: high dose haloperidol $\left(\mathrm{Hal} \mathrm{H}_{\mathrm{H}}\right)$ significantly increased stride length and maximum contact area compared to the saline condition (Sal). (B) Experiment 2 resulted in more typical parkinsonian symptoms; low dose haloperidol $\left(\mathrm{Hal}_{\llcorner}\right)$significantly increased stance duration and maximum contact area. (C) When comparing the haloperidol treated conditions from both experiments, high dose haloperidol increased base of support, maximum contact area and interlimb distance compared to the low dose condition. Data are means $\pm \mathrm{SEM}, \mathrm{n}=8$. Paired samples t-test differences were as follows: $\# p<0.05$, \#\# $p<0.01$. Please click here to view a larger version of this figure. 
A

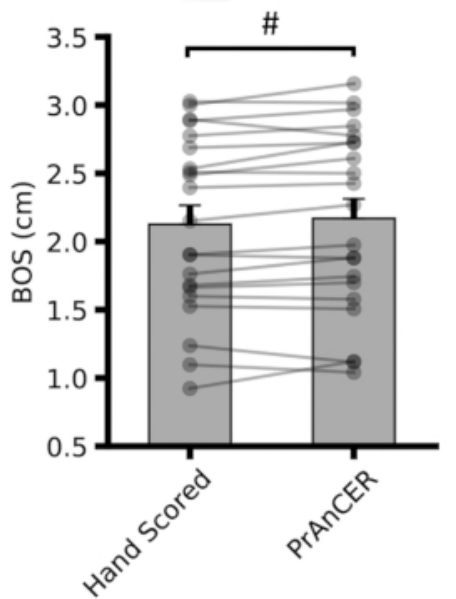

B

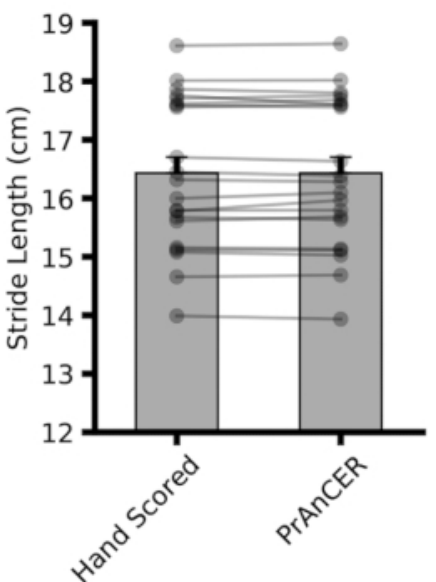

Figure 7. Accuracy of automated analysis. (A) The automated system significantly differs from manual scoring when measuring BOS, though this may be due to variations in manual centroid selection rather than detection errors. (B) The automated system is not significantly different from manual scoring for stride length. These accuracy results are consistent with those from other available systems. Data are means \pm SEM, $n$ $=21$. Paired samples t-test differences were as follows: $\# p<0.05$. Please click here to view a larger version of this figure.

A

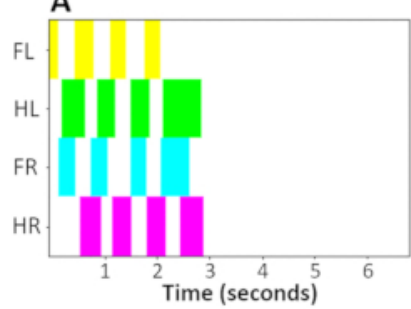

B

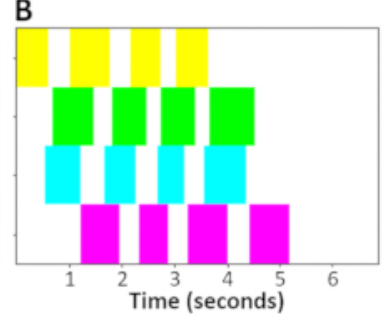

C

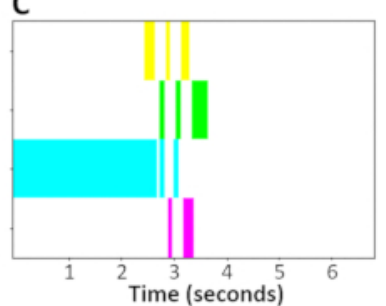

Figure 8. Comparison of temporal parameters. Temporal gait patterns for an animal treated with saline (A) and low dose haloperidol (B). (C) An illustration of the bracing-escape response from a rat given high dose haloperidol. As in Figure 5, colored blocks indicate when the paw was in contact with the ground (stance phase) and the white spaces indicate when the paw was in the air (swing phase). Abbreviations: FL, front left; $H L$, hind left; $F R$, front right; $H R$, hind right. Please click here to view a larger version of this figure.

\begin{tabular}{|c|c|c|c|c|c|c|c|c|}
\hline & \multicolumn{4}{|c|}{ Experiment 1} & \multicolumn{4}{|c|}{ Experiment 2} \\
\hline & Test 1 & Test 2 & Test 3 & Test 4 & Test 5 & Test 6 & Test 7 & Test 8 \\
\hline Group A & $\mathrm{Hal}_{\mathrm{H}}$ & Sal & $\mathrm{Hal}_{\mathrm{H}}$ & Sal & Sal & $\mathrm{Hal}_{\mathrm{L}}$ & Sal & $\mathrm{Hal}_{\mathrm{L}}$ \\
\hline Group B & Sal & $\mathrm{Hal}_{\mathrm{H}}$ & Sal & $\mathrm{Hal}_{\mathrm{H}}$ & $\mathrm{Hal}_{\mathrm{L}}$ & Sal & $\mathrm{Hal}_{\mathrm{L}}$ & Sal \\
\hline
\end{tabular}

Table 1. Experimental design. This table illustrates the experimental design used in this study. We used a within-subject design in which every rat was tested in the high dose haloperidol $\left(\mathrm{Hal}_{\mathrm{H}}\right)$, low dose haloperidol $\left(\mathrm{Hal}_{\mathrm{L}}\right)$ and saline $(\mathrm{Sal})$ conditions. Rats were divided into two groups; testing was counterbalanced for time of day and order of condition. 


\begin{tabular}{|l|l|}
\hline Parameter & Definition \\
\hline Stride Length & Distance between successive contacts of the same paw \\
\hline Step Length & $\begin{array}{l}\text { Distance between successive contacts of contralateral front or hind } \\
\text { paws along the axis of the direction of motion }\end{array}$ \\
\hline Base of Support (BOS) & $\begin{array}{l}\text { Distance between successive contralateral front or hind paws } \\
\text { perpendicular to axis of the direction of motion }\end{array}$ \\
\hline Maximum Contact Area & The maximum detected area of a hind print \\
\hline Interlimb distance & Distance between ipsilateral front and hind paws \\
\hline Stance Duration & The length of time a paw was in contact with the ground \\
\hline Swing Duration & The length of time a paw was not on the ground \\
\hline Stance to Swing Ratio (SSR) & Stance duration/swing duration \\
\hline Discrete Speed & Stride length/(stance duration + swing duration) for a paw \\
\hline Average Speed & Average of discrete speeds in the period used in analysis \\
\hline Speed Variability & Percent change in discrete speeds during a run \\
\hline Run Speed & Time to cross the tunnel/length of tunnel \\
\hline
\end{tabular}

Table 2. Description of gait parameters. This table describes the most commonly used gait parameters; those used in this study are indicated in bold.

\section{Discussion}

In this study, we tested PrAnCER, a new automated gait analysis system that utilizes contrast-enhanced videos produced using a translucent floor to obscure the animal's body and to yield clearly defined paw prints for simple automated detection. PrAnCER accurately identifies paw prints and is sensitive to changes in motor function. We used PrAnCER to assess gait alterations in an acute haloperidol model of PD. Although haloperidol did not induce the expected motor deficits of a robust PD model, we were nonetheless able to demonstrate that PrAnCER can accurately detect changes in gait patterns. Finally, we quantified the accuracy of PrAnCER and demonstrated that its measurement of key gait parameters is comparable to that of manual scoring.

In both haloperidol treated conditions, we observed a high incidence of freezing behavior (akinesia) followed by an escape response of running or bounding forward. While akinesia has been observed at a similar dose $(0.25 \mathrm{mg} / \mathrm{kg})$ in several studies ${ }^{16,23}$, this bounding behavior is not consistent with typical Parkinsonian symptoms $s^{3,14,19,24}$. Interestingly, we found that high dose haloperidol treatment resulted in significantly increased stride length. This finding was initially surprising because other haloperidol models of PD have shown a decrease in stride length ${ }^{3,19}$. However, they make sense in light of the 'bracing-escape' behavior pattern described by De Ryck et al. (1980), who reported that rats run to escape after akinetic periods, and that high speed gaits such as running and bounding are associated with increased stride length ${ }^{4,25}$ (Figure 8C). High dose treatment also resulted in significantly increased maximum contact area of the hind paws. Low dose haloperidol treatment resulted in more characteristic PD gait alterations including a significant increase in stance duration and maximum contact area (Figure 8A-B). These results may be a reflection of the muscle rigidity associated with haloperidol-induced akinesia.

Despite the unusual bracing-escape behavior, we were able to demonstrate that PrAnCER can indeed detect alterations in gait. We showed that in the correct lighting conditions, a translucent floor can produce a highly contrasted and detailed image of the paws. In the present study, we made a transparent floor translucent by covering it with vellum. The same effect could be achieved by placing another translucent covering, such as Mylar, over a transparent floor. Alternatively, the floor itself could be translucent by using, for example, frosted plexiglass. The translucent floor and simple plexiglass walkway are inexpensive and can be constructed in an afternoon. Our edge-detection-based analysis system is resilient to many variations in the apparatus and offers adjustable thresholds to adapt the system to different setups, disease models, or smaller animals such as mice.

Some gait parameter analyses were altered from conventional formulas because of aspects of the walkway. For example, our method of calculating speed differs from other gait studies; the translucent floor combined with LED lighting obscures the view of the body, so it is not possible to track body position to calculate speed as is usually done. For this study, speed was calculated by dividing the distance travelled between two contacts of the same paw by the time from first contact to second contact. Of course, other formulas could be used. For example, if an overall measure of speed is needed, one could divide the distance from the average of forepaw locations at the beginning and end of the run by the duration of the run.

Our analysis confirms that, while not identical to manual scoring, our automated system performs with high accuracy and generates reliable measures of gait. The apparatus described here was optimized for a simple, low-cost analysis of motor function. However, several alterations could be made that would expand the usefulness of PrAnCER. One limitation of our system is that the semitransparent floor, while allowing excellent paw detection, obscures the animals' body axis. Although we have not found it necessary, this could be addressed by adding an overhead camera to the system. Another improvement would be the use of a video camera with a higher frame rate. While we were able to obtain consistent estimates of temporal parameters, the accuracy of these measures is compromised at frame rates below $100 \mathrm{fps}{ }^{8}$. Adding a high-speed video camera would require no alteration of the analysis software while increasing the accuracy and precision of temporal measures. Additionally, several other gait systems use a mirror to simultaneously record the lateral and ventral views of the rat ${ }^{2,8,13}$. Adding this feature to our apparatus would permit more accurate quantification of speed and better observation of behavior during runs. 
In this study, we showed that the use of a semitransparent floor effectively isolates paw prints by blocking the visibility of objects not in contact with the walkway floor. We developed an automated scoring system that takes advantage of this high contrast paw print to accurately identify paws. We showed that this system, PrAnCER, quantified gait parameters with an accuracy comparable to commercial systems. We determined that administration of a high dose of haloperidol increased stride length and maximum contact area compared to saline. While this change is the opposite of what we expected, further review of existing literature indicates that it is likely part of the escape behavior observed in response to acute administration of haloperidol. Low dose haloperidol treatment resulted in more typical PD symptoms such as increased stance duration and maximum contact area. We conclude that while acute high dose haloperidol administration is a poor model to study gait impairments associated with PD, our study nonetheless demonstrated the ability of PrAnCER to accurately detect changes in motor function. In the future, we hope to further validate PrAnCER by studying the locomotor changes in other disease models.

\section{Disclosures}

The authors have no conflicts of interest to disclose.

\section{Acknowledgments}

This work was supported by the Carney Institute for Brain Science at Brown University.

\section{References}

1. Lakes, E. H., Allen, K. D. Gait analysis methods for rodent models of arthritic disorders: reviews and recommendations. Osteoarthritis and Cartilage. 24(11), 1837-1849, (2016).

2. Lee, H.Y., Hsieh, T.H., Liang, J.I., Yeh, M.L., \& Chen, J. J. Quantitative video-based gait pattern analysis for hemiparkinsonian rats. Medical \& Biological Engineering \& Computing. 50(9), 937-946, (2012).

3. Zhou, M., et al. Gait analysis in three different 6-hydroxydopamine rat models of Parkinson's disease. Neuroscience Letters. 584, 184-189, (2015).

4. Batka, R. J., Brown, T. J., Mcmillan, K. P., Meadows, R. M., Jones, K. J., Haulcomb, M. M. The need for speed in rodent locomotion analyses. Anatomical Record. 297(10), 1839-1864, (2014).

5. Guillot, T. S., Asress, S. A., Richardson, J. R., Glass, J. D., Miller, G. W. Treadmill gait analysis does not detect motor deficits in animal models of Parkinson's disease or amyotrophic lateral sclerosis. Journal of Motor Behavior. 40(6), 568-577, (2008).

6. Williams, M. T., et al. Kaolin-induced ventriculomegaly at weaning produces long-term learning, memory, and motor deficits in rats. International Journal of Developmental Neuroscience. 35, 7-15, (2014).

7. Hamers, F. P. T., Koopmans, G. C., Joosten, E. A. J. CatWalk-assisted gait analysis in the assessment of spinal cord injury. Journal of Neurotrauma,. 23(3-4), 537-548, (2006).

8. Kloefkorn, H. E., et al. Automated Gait Analysis Through Hues and Areas (AGATHA): A Method to Characterize the Spatiotemporal Pattern of Rat Gait. Annals of Biomedical Engineering. 45(3), 711-725, (2017).

9. Pereira, J. E., et al. A comparison analysis of hindlimb kinematics during overground and treadmill locomotion in rats. Behavioural Brain Research. 172(2), 212-218, (2006).

10. Nakamura, A., et al. Low-cost three-dimensional gait analysis system for mice with an infrared depth sensor. Neuroscience Research. 100, 55-62, (2015).

11. Hamers, F. P., Lankhorst, A. J., van Laar, T. J., Veldhuis, W. B., Gispen, W. H. Automated quantitative gait analysis during overground locomotion in the rat: its application to spinal cord contusion and transection injuries. Journal of Neurotrauma. 18(2), 187-201, (2001).

12. Mendes, C. S., Bartos, I., Márka, Z., Akay, T., Márka, S., Mann, R. S. Quantification of gait parameters in freely walking rodents. BMC Biology. 13(50), (2015).

13. Machado, A. S., Darmohray, D. M., Fayad, J., Marques, H. G., Carey, M. R. A quantitative framework for whole-body coordination reveals specific deficits in freely walking ataxic mice. eLife. 4, (2015).

14. Duty, S., \& Jenner, P. Animal models of Parkinson's disease: a source of novel treatments and clues to the cause of the disease. British Journal of Pharmacology. 164(4), 1357-1391, (2011).

15. Kulkarni, S. K., Bishnoi, M., Chopra, K. In vivo microdialysis studies of striatal level of neurotransmitters after haloperidol and chlorpromazine administration. Indian Journal of Experimental Biology. 47(2), 91-97, (2009).

16. Jain, N. S., Tandi, L., Verma, L. Contribution of the central histaminergic transmission in the cataleptic and neuroleptic effects of haloperidol. Pharmacology Biochemistry and Behavior. 139(Part A), 59-66, (2015).

17. Steinpreis, R. E., Anders, K. A., Branda, E. M., Kruschel, C. K. The Effects of Atypical Antipsychotics and Phencyclidine (PCP) on Rotorod Performance. Pharmacology Biochemistry and Behavior. 63(3), 387-394, (1999).

18. Baptista, P. P. A., et al. Physical exercise down-regulated locomotor side effects induced by haloperidol treatment in Wistar rats. Pharmacology, Biochemistry, and Behavior. 104, 113-118, (2013).

19. Chuang, C.S., Su, H.L., Cheng, F.C., Hsu, S., Chuang, C.F., Liu, C.S. Quantitative evaluation of motor function before and after engraftment of dopaminergic neurons in a rat model of Parkinson's disease. Journal of Biomedical Science. 17(9), (2010).

20. Bugalho, P., Alves, L., Miguel, R. Gait dysfunction in Parkinson's disease and normal pressure hydrocephalus: a comparative study. Journal of Neural Transmission. 120(8), 1201-1207, (2013).

21. Bradski, G. The OpenCV Library. (2000).

22. Chen, H., Du, J., Zhang, Y., Barnes, K., Jia, X. Establishing a Reliable Gait Evaluation Method for Rodent Studies. Journal of Neuroscience Methods. 283, 92-100, (2017).

23. De Ryck, M., Hruska, R. E., Silbergeld, E. K. Estrogen and haloperidol-induced versus handling-related catalepsy in male rats. Pharmacology, Biochemistry, and Behavior. 17(5), 1027-1035, (1982).

24. Bellardita, C., Kiehn, O. Phenotypic Characterization of Speed-Associated Gait Changes in Mice Reveals Modular Organization of Locomotor Networks. Current Biology. 25(11), 1426-1436, (2015). 
25. De Ryck, M., Schallert, T., Teitelbaum, P. Morphine versus haloperidol catalepsy in the rat: a behavioral analysis of postural support mechanisms. Brain Research. 201(1), 143-172. (1980). 